OPEN ACCESS

Edited by: Ana Preto,

University of Minho, Portugal

Reviewed by: Cinzia Domenicotti, University of Genoa, Italy Barbara Marengo,

University of Genoa, Italy

*Correspondence:

Boutaina Daher

bdaher@centrescientifique.mc Jacques Pouysségur

pouysseg@unice.fr

Specialty section: This article was submitted to

Cancer Metabolism, a section of the journal Frontiers in Oncology

Received: 21 February 2020 Accepted: 16 April 2020 Published: 07 May 2020

Citation:

Daher B, Vučetić M and Pouysségur J (2020) Cysteine Depletion, a Key Action to Challenge Cancer Cells to Ferroptotic Cell Death Front. Oncol. 10:723. doi: 10.3389/fonc. 2020.00723

\section{Cysteine Depletion, a Key Action to Challenge Cancer Cells to Ferroptotic Cell Death}

\author{
Boutaina Daher ${ }^{1 *}$, Milica Vučetić ${ }^{1}$ and Jacques Pouysségur ${ }^{1,2 *}$ \\ ${ }^{1}$ Medical Biology Department, Centre Scientifique de Monaco (CSM), Monaco, Monaco, ${ }^{2}$ Institute for Research on Cancer \\ and Aging (IRCAN), CNRS, INSERM, Centre A. Lacassagne, Université Côte d'Azur, Nice, France
}

Cancer cells are characterized as highly proliferative at the expense of enhancement of metabolic rate. Consequently, cancer cells rely on antioxidant defenses to overcome the associated increased production of reactive oxygen species (ROS). The reliance of tumor metabolism on amino acids, especially amino acid transport systems, has been extensively studied over the past decade. Although cysteine is the least abundant amino acid in the cell, evidences described it as one of the most important amino acid for cell survival and growth. Regarding its multi-functionality as a nutrient, protein folding, and major component for redox balance due to its involvement in glutathione synthesis, disruption of cysteine homeostasis appears to be promising strategy for induction of cancer cell death. Ten years ago, ferroptosis, a new form of non-apoptotic cell death, has been described as a result of cysteine insufficiency leading to a collapse of intracellular glutathione level. In the present review, we summarized the metabolic networks involving the amino acid cysteine in cancer and ferroptosis and we focused on describing the recently discovered glutathione-independent pathway, a potential player in cancer ferroptosis resistance. Then, we discuss the implication of cysteine as key player in ferroptosis as a precursor for glutathione first, but also as metabolic precursor in glutathione-independent ferroptosis axis.

\section{Keywords: xCT transporter, cysteine, lipid peroxides, glutathione, ferroptosis, tumor-resistance}

\section{INTRODUCTION}

Since the early 20th century, the reprogramming of cellular metabolism has been recognized as one of the major hallmarks of oncogenesis (1) that has great potential for anti-cancer treatment. Key signatures of cancer cells, due to their highly proliferative nature, are intensified metabolic rate, increased oxidative pressure, and consequently, reliance on antioxidant defense in term of redox homeostasis maintenance. The central role of amino acids in antioxidant defense is mainly due to the involvement of serine, glutamine/glutamate and cysteine, in glutathione (GSH) and nicotinamide adenine dinucleotide phosphate (NADPH) production $(2,3)$. One particularity of cysteine is its semi-essential nature and its implication in GSH homeostasis. GSH is a key non-enzymatic player in cellular antioxidant defense and is broadly implicated in tumor initiation, progression, metastasis, and resistance. Nutrient supply and redox balance are therefore intertwined and of great importance for anti-cancer treatment. In the present review, we will 
describe in more details cysteine implication in cancer (patho)physiology from two aspects; cysteine as a proteinogenic amino acid and cysteine as an amino acid involved in the GSHand thus redox- homeostasis.

\section{CYSTEINE, A KEY PLAYER IN TUMOR METABOLISM}

Cysteine is a sulfur-containing amino acid. Even though it is described to be a "non-essential" amino acid, in conditions of high nutrient demands, it becomes essential. In the liver, a particular metabolic pathway called transsulfuration permits the supply of cysteine by conversion of an essential amino acid: methionine. Yet, this amino acid interconversion is insufficient to provide the cysteine requirements of rapidly dividing cancer cells (4). As mentioned previously, cysteine is a thiol-containing amino acid, which nucleophilicity makes it highly susceptible to redox changes. Notably, impressive complexity of the cysteinome dynamic reflects its important role in the cell. Indeed cysteine has a crucial role in many processes such as assembly, protein folding stability and trafficking, biosynthesis of coenzyme A and taurine, iron-sulfur (Fe-S) cluster biogenesis, detoxification of heavy metals and redox balance (5). A number of pathologies have been characterized by an unbalanced cysteinome profile, including cystinuria, renal calculi, Huntington's disease, and Alzheimer's disease (6-8). In cancer, the implication of cysteine in tumor formation, propagation and resistance has been widely described (5).

The building block for three essential nutrients (carbohydrates, lipids, and proteins): simple sugars, fatty and amino acids, are provided from diet. Contrary to for example fatty acids, amino acids due to their lipophobicity require transporters for the import/export. Up to now, more than 30 different amino acid transporters have been described in mammalian cells, however, a small, co-called "minimal set" among them is consistently overexpressed in many different tumor types (9-11). These transporters are LAT1, ASCT2 and the $\mathrm{Xc}^{-}$system. According to our previous study, LAT1 (standing for L-type Amino acid Transporter 1) is indispensable for transport of essential amino acids, general amino acid homeostasis, and consequently, tumor growth (12). ASCT2 or Alanine-Serine-Cysteine Transporter 2 is a transporter that exchanges small neutral amino acids and plays a crucial role in glutamine uptake and the promotion of tumor growth, independently of LAT1 activity (13). The third overexpressed transporter in cancer is the $\mathrm{X}_{\mathrm{c}}^{-}$system, an exchanger that imports cystine, the oxidized form of cysteine, and exports glutamate. This sodium-independent antiporter is composed of two subunits: xCT (gene name SLC7A11), a subunit responsible for the amino acid exchange, and a chaperone CD98 (gene name SLC3A2). In 2011, the transmembrane glycoprotein CD44, a cancer stem-like cell marker, and more precisely the CD44 variant $\left(\mathrm{CD} 44^{\mathrm{v}}\right)$ capable to bind hyaluronan has also been described to interacts and stabilizes $\mathrm{X}_{\mathrm{c}}^{-}$system (14) (Figure 1). Although the role of CD44 in the transport activity of xCT has not been validated so far, an interesting implication in iron endocytosis via CD44-bound hyaluronates is proposed (15) (Figure 1). Our group recently described that a genetic disruption of the xCT subunit using CRISPR-Cas9 inhibits protein synthesis and proliferation in vitro (16) and leads to a specific non-apoptotic cell death named ferroptosis, that will be described later in this review. A ${ }^{14} \mathrm{C}$-cystine transport assay in $\mathrm{xCT}$ knockout (xCT-KO) cells revealed this transporter as unique and indispensible for cystine uptake, as a complete abolishment of cystine transport has been observed. In contrast, in in vivo assay, $\mathrm{xCT}-\mathrm{KO}$ pancreatic ductal adenocarcinoma (PDAC) cells injected subcutaneously managed to form a tumor, although with a short delay. This indicates that other mechanisms are involved in the maintenance of intracellular cysteine pool in vivo allowing tumor growth. Indeed, one of the poorly discussed limits of cystine transport study in vitro is the fact that the commonly used culture media contains exclusively oxidized form of cysteine. Consistent with this, use of a reducing source such as $\beta$-mercaptoethanol allows reversal of xCT-KO phenotype, as it has been reported couple decades ago by Bannai's group $(17,18)$. Therefore, highly dynamic ratio of cystine/cysteine couple in vivo can explain the discrepancy with in vitro phenotype. Transport of reduced form of cysteine has been assigned to the transporters form ASCT family. However, in case of the ASCT2, studies showed that cysteine is actually a competitive inhibitor and not a substrate for ASCT2 $(19,20)$. Similarly, preliminary results in our group indicate that ASCT2 is not involved in cysteine uptake in surviving $\mathrm{xCT}-\mathrm{ASCT} 2$ double knockout PDAC cells in presence of $\beta$ mercaptoethanol. Our laboratory at the moment is focused on the examination of this highly elusive transport system for the import of cysteine.

The highly conserved mechanistic target of rapamycin (mTOR) regulates protein synthesis, metabolism and growth. Activation of the mTOR complex 1 (mTORC1) relies not only on insulin and growth factors activating, respectively, PI3K and ERK1/2, but also on amino acids. Indeed translocation of mTORC1 from the cytoplasm to the lysosome, a rich compartment in amino acids, is critical for mTORC1 activation (21). In addition the specific activation of mTORC1 by the amino acids glutamine, arginine and leucine is well-described $(21,22)$. Interestingly, recent report suggested that cysteine per $s e$ is also able to regulate mTORC1 activity (23). In line with this, disruption of cystine uptake inhibits mTORC1 activation, leading to an inhibition of protein synthesis $(16,24)$. It is interesting to note that the capacity of sensing amino acids has been achieved by different mechanisms so that intracellular protein synthesis homeostasis is ensured with high fidelity. Except mTORC1, another important pathway in this regard is amino acid-starvation pathway. Namely, the protein kinase General Control Nonderepressible (GCN2) is a sensor of amino acids that is activated by the intracellular accumulation of uncharged tRNA $(25,26)$. GCN2 represses general protein synthesis and activates the transcription of genes involved in the synthesis and transport of amino acids via activation of ATF4 transcriptional factor. This GCN2-ATF4 pathway is crucial for tumor cell survival during nutrient deprivation (27). Our data from PDAC cell lines showed that genetic ablation of 


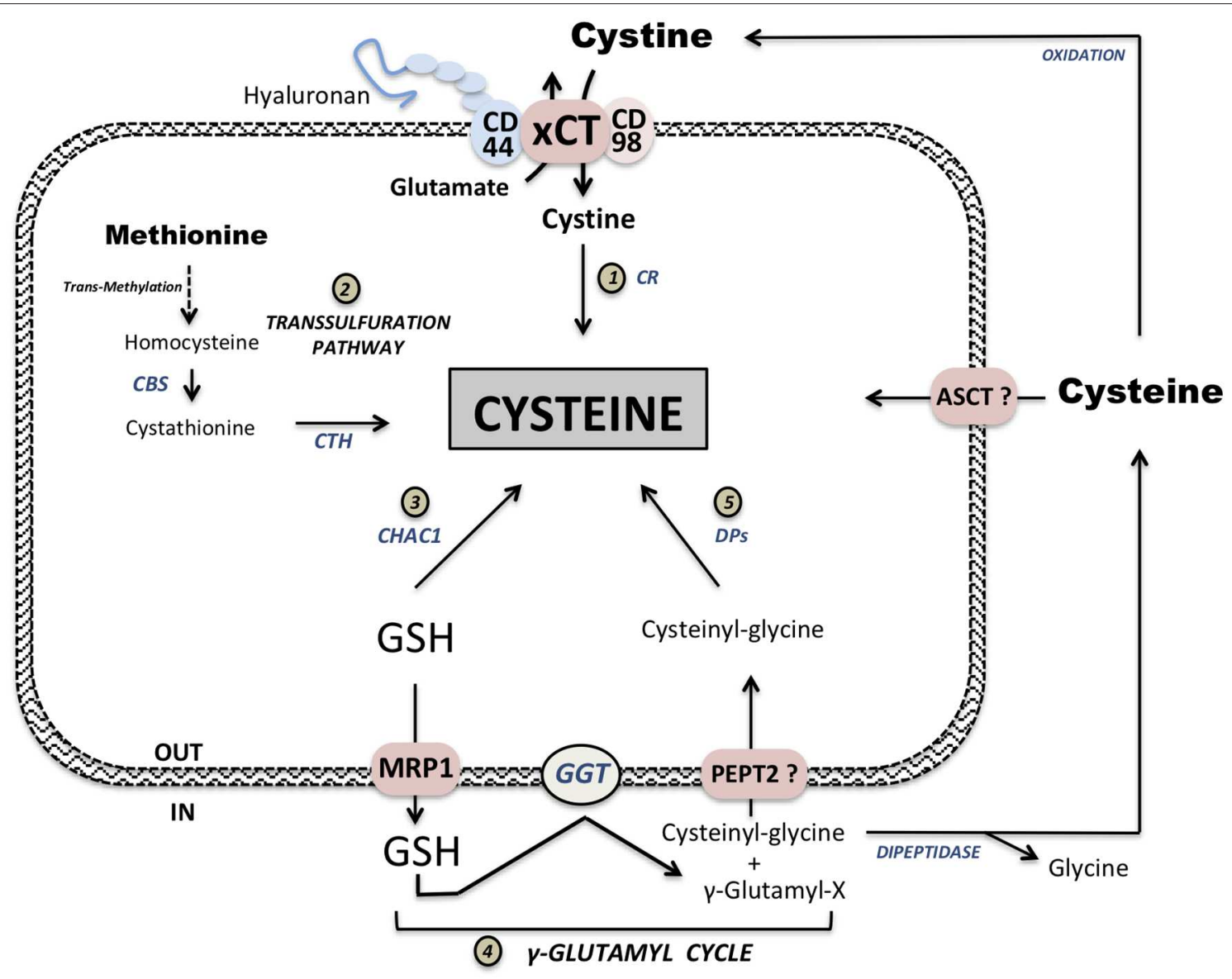

FIGURE 1 | Intracellular cysteine pool supply. Extracellular oxidized cystine is imported at the expense of one glutamate molecule via $\mathrm{XC}^{-}$system composed of two subunits: $\mathrm{xCT}$ transporter and the chaperone CD98. This complex XCT is also associated with the stem-like cancer cell marker CD44v. Imported cystine is then reduced to cysteine by cystine reductase (CR) (1). Methionine conversion leads to cysteine synthesis via the transsulfuration pathway (2). Two important steps in this synthesis are conversion from homocysteine to cystathionine by cystathionine $\beta$-synthase (CBS) and synthesis of cysteine from cystathionine by cystathionase (CTH). Degradation of glutathione (GSH) via CHAC1 intracellularly provides cysteine supply (3). GSH, either from exogenous sources or exported from cells via Multidrug Resistance Protein 1 exporter (MRP1), is cleaved extracellularly by $\gamma$-Glutamyl transferase (GGT) forming $\gamma$-Glutamyl-X substrate and Cysteinyl-Glycine. This Cysteinyl-Glycine dipeptide can either be potentially transported via PEPT2 or cleave by dipeptidase releasing cysteine and glycine (5). $\gamma$-Glutamyl moiety can be complexed to available extracellular cyst(e)ine forming $\gamma$-Glutamyl-cysteine. Cysteine supply from GSH is one of the main function of $\gamma$-Glutamyl-cycle (4). Available extracellular cysteine is then transported via ASCT family members but can also be oxidized and imported via xCT.

$\mathrm{xCT}$ transporter leads to intracellular cysteine deficiency, and therefore GCN2-ATF4 pathway activation (16). The results described in PDAC cells have also been confirmed in human breast cancer after genetic or pharmacologic inhibition of this transporter (28).

In brief, activation of the GCN2-ATF4 amino acid stress pathway and inhibition of protein synthesis through inhibition of mTORC1 demonstrates the strong proteogenic role played by cysteine in tumor cells. Nevertheless, the role of building the protein molecules is not the only function of cysteine. The particularity of this amino acid is its bifunctionality, and its other essential role is the building up of cellular antioxidant defenses via the biosynthesis of the most conserved and abundant non-enzymatic antioxidant in the cell: glutathione.

\section{GLUTATHIONE: HOMEOSTASIS AND FUNCTIONS}

Glutathione $(\mathrm{GSH})$ is the most abundant non-protein thiol in mammalian cells, reaching an intracellular concentration in $\mathrm{mM}$ range, whereas its plasma concentration does not exceed micromolar range. In the cell, $90 \%$ of GSH is located in the cytoplasm, $10-12 \%$ in the mitochondria, and a small percentage in the endoplasmic reticulum (ER) (29). This small tripeptide is composed of glutamate, cysteine, and glycine. Its biosynthesis is a two-step enzymatic cascade, including first a specific $\gamma$-ligation of glutamate and cysteine by $\gamma$-glutamate-cysteine ligase (GCL) and then the formation of peptide bond between this dipeptide and glycine by glutathione synthetase (GS). The GCL enzyme consists of two subunits, a heavy catalytic subunit, GCLc, and 


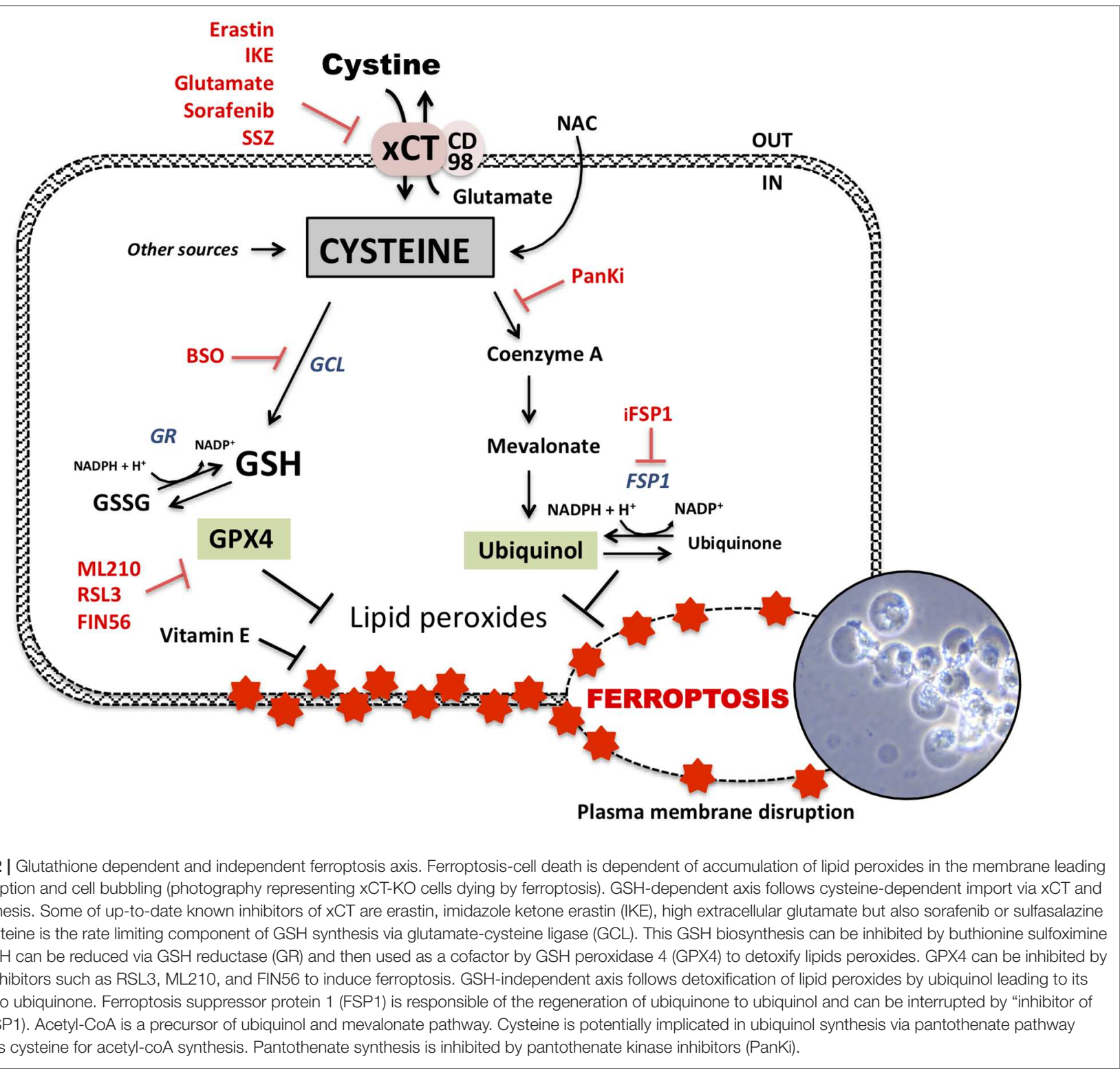

a light regulatory subunit, GCLm. Cysteine availability is the limiting factor of GSH synthesis due to the fact that GCLc Km for cysteine, around $270 \mu \mathrm{M}$, is roughly equal to its intracellular concentration. GSH is involved in many important cellular functions via its key role in antioxidant defense, protecting the cell against free radicals produced as metabolic by-products, either directly or indirectly. Numerous studies demonstrated that this small molecule is crucial in many different human diseases such as aging, diabetes, acquired immune deficiency syndrome (AIDS), as well as neurodegenerative and liver diseases (30). The importance of glutathione in tumor metabolism and particularly in resistance mechanisms has been widely studied during the last decades. One of the well-described roles played by GSH is the detoxification of xenobiotics such as different drugs, and thus it is fundamental for the resistance to chemo-, but also radiotherapy. Indeed, multidrug and radiation resistance in tumor cells have been associated with higher intracellular levels of GSH, and increased level of GSH is a poor prognostic factor in many types of cancer (31).

The $\gamma$-glutamatyl cycle or Meister cycle initially proposed in the 60 s described the synthesis and breakdown of GSH, making it a strong cysteine donor in physiological and pathological conditions (32). Cysteine synthesis via the transsulfuration pathway and GSH biosynthesis occurs primarily in the liver in physiological conditions, while in the pathology other cells can take over the same role or contribute to it (developed later in next paragraph). GSH excreted in the blood is cleaved, to its constituents; and de novo synthesis of GSH by cancer cells occurs as follows: GSH is first exported from the cell of origin via transporters known as Multiresistance Drug Proteins (MRPs), which belongs to the ATP binding cassette (ABC)s transporter family and is well-known player in cancer resistance 
mechanisms (33). Then, once in the extracellular space, GSH is cleaved by $\gamma$-Glutamyl-Transferase (GGT), which is also known as a poor prognostic factor for cancer patients (34). With an active site at the external surface of the plasma membrane, GGT catalyzes the transfer of $\gamma$-glutamatyl moiety from GSH to free amino acid, and thereby, released cysteineglycine dipeptide that can be transported in its intact form via the proton-coupled oligopeptide transporter family member PEPT2 $(35,36)$ or further cleaved by dipeptidases to cysteine and glycine. On the other side, the $\gamma$-glutamyl-amino acids are converted into 5-oxoproline and the corresponding amino acid by $\gamma$-glutamyl cyclotransferase. One interesting possibility is that $\gamma$-glutamyl can be complexed to extracellular available free cyst(e)ine, imported into the cell and as such can serve as a substrate for GS during GSH synthesis (bypassing GCL reaction). In physiological conditions, oxidized GSH can be recycled intracellularly by GSH reductase (GR) using NADPH as a reducing power. However, in stressful, oxidative-compromising conditions, this reductase seems not to be sufficient, underlying the importance of this $\gamma$-glutamyl cycle (37). As described by Bannai, this recycling cycle provides very reliable source of cysteine, and thus GSH, to the cells. Therefore, GGT localization across the membrane permits direct uptake of the extracellular reduced form of cysteine before its oxidation (38). However, whether intact GSH can cross the cellular membrane via a specific transporter remains unclear (39). Further investigation of the pathways involving GSH is expected to bring important insights into cancer (patho)physiology understanding (2).

Besides ATF4, another transcription factor that regulates $\mathrm{xCT}$ expression is the nuclear factor erythroid 2-related factor 2 (NRF2) via the antioxidant response element (ARE) present in the promoter region of xCT gene $(40,41)$. GSH levels directly correlate with cysteine availability; therefore, a disruption of cysteine uptake, either genetically or chemically, efficiently depletes GSH intracellular levels $(16,42)$. As described previously, GSH has multiple roles in the cell and one of them is functioning as cofactor for the enzyme glutathione peroxidase $4(\mathrm{GPx} 4)$. This peroxidase, with a selenocysteine at its active site, converts lipid hydroperoxides to lipid alcohols using reducing power of GSH. Lipid peroxides can be produced either spontaneously or by enzyme-catalyzed processes. Freeradical chain reaction occurs in an oxidatively-compromised environment where ROS production overcomes their removal. In the specific case of cysteine-deprived cell death, lipid peroxidation acquires a character of chain-reaction due to the Fenton reaction with ferrous iron $\left(\mathrm{Fe}^{2+}\right)$. Namely, redoxactive metals, like $\mathrm{Fe}^{2+}$, react with peroxides generating highly active hydroxyl radicals $\left(\mathrm{R}-\mathrm{HO}{ }^{\bullet}\right)$ that further propagate the peroxidation reaction (43). Ferroptosis, coined by Stockwell's group in 2012, designates a specific non-apoptotic cell death caused by such accumulation of lipid peroxides following cystine deprivation (42). Disruption of cysteine uptake and collapse of intracellular GSH pool induces an inhibition of the detoxifying activity of GPx4 and excessive accumulation of oxidatively damaged lipids at the membrane, although it remains unclear if this solely affects the cell plasma membrane, or the effect also extends to organelle membranes. All in all, the different pathways involved in GSH homeostasis are an indication of the complex dynamic and quick turn over of this tripeptide, and provide clues for potential targets for a GSH-depleting, ferroptosis-inducing strategy.

\section{CYSTEINE, LIPID PEROXIDES AND FERROPTOSIS}

\section{Glutathione-Dependent Ferroptosis}

Since characterization of ferroptosis in 2012, the cysteine-GSHGPx4 axis is described as essential pathway for its regulation, and thus seen as potential therapeutic target. Up to now, powerful genetic tools allowed clarification of the significance, dispensability and potential of cystine transporters for ferroptotic cell death in PDAC and breast cancer $(16,28)$. On the other side, now there is growing interest in the development of specific pharmacological inhibitors of $\mathrm{xCT}$ that will prove fundamental research in the clinical settings. Almost 20 years ago, a high extracellular level of glutamate was described as inhibitor for cystine uptake and inductor of a specific cell death termed oxytosis (44). Indeed, in 1988 Bannai reported glutamine import via ASCT2 and conversion into glutamate that is exported in exchange of cystine import (1:1) via xCT (45). Yet, more recently, a metabolomic analysis reveals the importance of glutamine uptake by ASCT2 and its conversion into ROSproducing intermediate metabolite $\alpha$-Ketoglutarate as marker of sensitivity during sulfasalazine xCT-inhibition cell death in head and neck squamous cells carcinoma (46). Glutaminolysis was also reported to sensitize melanoma cells to ferroptosis (47). Therefore, those studies suggest that ASCT2 expression can be a marker of ferroptosis sensitivity.

A high extracellular glutamate concentration was one of the first xCT inhibitors described but a few years later, erastin (standing for eradicator of RAS and small T antigen-expressing cells) was identified by Stockwell's group in RAS-mutated cancer cell lines as inhibitor of xCT transport (42). Erastin is widely used in in vitro studies as efficient inducer of ferroptosis in many different cancer types, including breast, PDAC, lymphoma, renal, brain and ovarian cancers (48). A recent study has described a new form of erastin, imidazole ketone erastin (IKE), characterized as more metabolically stable than the previous form (49). Other small compounds are also described as ferroptosis inducers, including two FDA-approved drugs, sulfasalazine, prescribed for lung carcinoma and fibrosarcoma $(50,51)$ and sorafenib $(52)$. However, our team found that the specificity of those two compounds to $\mathrm{xCT}$ was relatively low, as addition of cysteine analog N-Acetyl-Cysteine (NAC), could not rescue cells from dying (16). Another possible way to induce depletion of intracellular cysteine has been proposed by Cramer's group using systemic depletion of cysteine in the plasma of leukemic mice. This was done by employing an engineered cyst(e)inase enzyme resulting in the suppression of tumor growth in breast and prostate cancer xenografts (53). Nevertheless, it is crucial to note that recently conversion of methionine to cysteine via transsulfuration pathway is a potential resistance mechanism under cysteine depletion conditions. The conversion 
from homocysteine to cystathionine by cystathionine $\beta$-synthase (CBS) is described to be a key player in restoring intracellular cysteine pool as decreasing its expression increases sensitivity to ferroptosis in ovarian cancer cells upon erastin treatment (54). Downstream reaction that forms cysteine from cystathionine by cystathionase $(\mathrm{CTH})$ has also been recently described to play a key role in adaptation mechanisms during cysteine-deprived induced stress in a wide variety of cancer cells lines (55). Investigating the molecular mechanisms involved in restoring the intracellular redox-buffer cysteine is of a great interest in the understanding of ferroptosis resistance mechanisms. The importance of this pathway is not only crucial for ferroptosis as cystathionase is also described to be involved in senescence evasion in melanocytes and melanoma cells (56).

However, other systems downstream of cystine uptake can also play an important role. For instance, GSH can be depleted, through inhibition of its synthesis using an inhibitor of GCL (buthionine sulfoximine, BSO) (57) under conditions that maintain the intracellular cysteine pool intact,. Another strategy to deplete GSH is increasing its efflux. Dixon's group demonstrated that the GSH exporter MRP1 sensitizes HPA1 erastin-treated cells to ferroptosis and concordantly, MRP1 inhibition leads to a retention of GSH and leads to ferroptosis resistance (58). Furthermore, another recently described player involved in GSH catabolism is the specific cytoplasmic GSH-degrading enzyme CHAC1 (59). Interestingly, this enzyme enhances cysteine starvation-induced ferroptosis through activation of the GCN2-eIF2 $\alpha$-ATF4 pathway in human triple negative breast cancer cells (60). The implication of CHAC1 in increasing sensitivity to ferroptosis was recently confirmed in Burkitt's lymphoma during artesunate-induced ferroptosis (61). Those two mechanisms involved in efflux and intracellular GSH degradation can be of great interest to challenge ferroptosisresistant cells.

The third major target is the selenoenzyme GPx4. Different inhibitors of GPx4 induce ferroptosis such as RLS3, FIN56, and ML210 (24) and more recently, resibufugenin (62). Postchemotherapy-"persister" cells resistant to lapatinib treatment in breast, melanoma, lung, and ovarian cancer have been characterized as GPx4-dependent (63). Therefore, making ferroptosis inducers druggable for cancer therapy sounds like a promising strategy to challenge and overcome acquired resistance to other drugs. In theory, targeting any node of cysteine-GSH-GPx4 axis seems to be sufficient to induce ferroptosis. Importantly, results obtained from knockout mice suggests that $\mathrm{xCT}$ inhibition could have the least off-side effect in comparison with the GPx4 and GCL $(18,64,65)$. Indeed, $\mathrm{xCT}^{-/-}$ mice are healthy and fertile despite an increase in cystine plasma concentration and a decrease in GSH plasma level. Therefore, developing an efficient and specific xCT inhibitor is a promise of great advance in cancer therapy.

\section{Glutathione-Independent Ferroptosis}

Although described since the beginning to be the main actor of ferroptosis inhibition, it has been recently described that the cysteine-GSH-GPx4 axis can be, at least in part, dispensable. A recent genetic screen of genes complementing the loss of GPx4 in resistant cell lines uncovered new players for ferroptosis inhibition. A specific oxidoreductase, previously known as apoptosis-inducing-factor mitochondrial-2 (AIFM2), capable of recycling reduced ubiquinol (Co-enzymeQ ${ }_{10} \mathrm{H}_{2}$ ) from ubiquinone at the expense of $\mathrm{NAD}(\mathrm{P}) \mathrm{H}$, has been presented as a potential ferroptosis inhibitor due to the fact that its overexpression complements the loss of GPx4 in PFA1 and human fibrosarcoma $(66,67)$. Therefore, since then, this AIFM2 oxidoreductase has been re-named to Ferroptosis Suppressor Protein-1 (FSP1) (Figure 2). Those compensatory mechanisms depend on the $\mathrm{NAD}(\mathrm{P}) \mathrm{H}$-mevalonate pathway that synthesize ubiquinol. Ubiquinol traps radicals undergoing lipid peroxidation in the membrane. Therefore, the discovery of this parallel GSH-independent mechanism for lipid peroxide scavenging is of great interest for development of ferroptosisbased potential chemotherapeutics. Finally, membrane lipid composition and more importantly the long polyunsaturated fatty acid (PUFA) is playing a key role in ferroptosis sensitivity. This PUFA membrane enrichment is triggered by the specific enzyme acyl-CoA synthetase long-chain family member 4 (ACSL4). Interestingly ACSL4 was preferentially expressed in a panel of basal-like breast cancer cell lines and predicted their sensitivity to ferroptosis (68).

\section{CONCLUSION AND REMAINING QUESTIONS}

During past decade, investigation of ferroptosis from both aspects: induction and prevention, has become a topic of interests for numerous different pathologies. Nevertheless, a remaining undiscussed point is the role of cysteine in ferroptosis independently from GSH synthesis. In other words, how similar are the phenotypes of cysteine-depleted vs. GSH-depleted cells? Is ferroptosis caused exclusively by an excessive lipid peroxides accumulation due to GSH depletion and oxidative damage, or also by cysteine insufficiency itself? To investigate the role played by this amino acid, labeled cystine was used to follow its incorporation in PDAC cells before and after treatment with IKE. As described previously, the major part of exogenous cystine is incorporated in GSH, yet unexpectedly, the remaining part is incorporated in co-enzyme A synthesis via the pantothenate pathway (69). Co-enzyme A is a precursor of cholesterol and coenzyme Q10 (ubiquinol) a product of the mevalonate pathway, but also a key player in fatty acid biosynthesis and $\beta$-oxidation. Notably, lipid metabolism plays a crucial role in ferroptosis (70). We therefore suggest an additional role of cysteine, independent of GSH synthesis, in the prevention of ferroptosis. On the other hand, inhibition of GSH synthesis with BSO, independently of the cysteine pool, has been repeatedly described to induce ferroptosis (42). In contrast, many recent studies explored and demonstrated the implication of GSH depletion in induction of apoptosis via depletion of mitochondrial GSH pool leading to the release of cytochrome $c$, when combined with chemotherapy in breast cancer and leukemia, respectively $(71,72)$. Moreover, GSH has also been involved in other types of cell death such as necroptosis and for more details, refer to Lv's review (73). 
In line with this, one other mechanism recently described to be involved in the resistance to GSH depletion is the overexpression of deubiquitinases that inhibit protein degradation following ER-stress (74). This system, independent of lipid peroxide and apoptotic-mitochondrial defect, reveals the complexity of GSHdepletion-induced cell death pathways. Our team is currently validating this hypothesis using knockout cell lines specifically deficient for GSH synthesis. All in all, despite recent progress in the field, the detailed mechanisms of ferroptosis are still largely unknown and a significant amount of research remains to be developed in this new exciting area of research.

\section{REFERENCES}

1. Hanahan D, Weinberg RA. Hallmarks of cancer: the next generation. Cell. (2011) 144:646-74. doi: 10.1016/j.cell.2011.02.013

2. Toledano MB, Huang M-E. The unfinished puzzle of glutathione physiological functions, an old molecule that still retains many enigmas. Antioxid Redox Signal. (2017) 27:1127-9. doi: 10.1089/ars.2017.7230

3. Vučetić M, Cormerais Y, Parks SK, Pouysségur J. The central role of amino acids in cancer redox homeostasis: vulnerability points of the cancer redox code. Front Oncol. (2017) 7:319. doi: 10.3389/fonc.2017.00319

4. Pajares MA, Pérez-Sala D. Mammalian sulfur amino acid metabolism: a nexus between redox regulation, nutrition, epigenetics, and detoxification. Antioxid Redox Signal. (2018) 29:408-52. doi: 10.1089/ars.2017.7237

5. Combs JA, DeNicola GM. The non-essential amino acid cysteine becomes essential for tumor proliferation and survival. Cancers (Basel). (2019) 11:678. doi: $10.3390 /$ cancers 11050678

6. Sahota A, Tischfield JA, Goldfarb DS, Ward MD, Hu L. Cystinuria: genetic aspects, mouse models, and a new approach to therapy. Urolithiasis. (2019) 47:57-6. doi: 10.1007/s00240-018-1101-7

7. Kumar A, Ratan RR. Oxidative stress and huntington's disease: the good, the bad, and the ugly. J Huntingtons Dis. (2016) 5:217-37. doi: $10.3233 /$ JHD-160205

8. Gu L, Robinson RAS. A simple isotopic labeling method to study cysteine oxidation in alzheimer's disease: oxidized cysteine-selective dimethylation (OxcysDML). Anal Bioanal Chem. (2016) 408:2993-3004. doi: 10.1007/s00216-016-9307-4

9. Kandasamy P, Gyimesi G, Kanai Y, Hediger MA. Amino acid transporters revisited: new views in health and disease. Trends Biochem Sci. (2018) 43:75289. doi: 10.1016/j.tibs.2018.05.003

10. Barretina J, Caponigro G, Stransky N, Venkatesan K, Margolin AA, Kim S, et al. The cancer cell line encyclopedia enables predictive modelling of anticancer drug sensitivity. Nature. (2012) 483:603-7. doi: 10.1038 /nature11003

11. McCracken AN, Edinger AL. Nutrient transporters: the achilles' heel of anabolism. Trends Endocrinol Metab. (2013) 24:200-8. doi: 10.1016/j.tem.2013.01.002

12. Cormerais Y, Giuliano S, LeFloch R, Front B, Durivault J, Tambutte E, et al. Genetic disruption of the multifunctional CD98/LAT1 complex demonstrates the key role of essential amino acid transport in the control of mTORC1 and tumor growth. Cancer Res. (2016) 76:4481-92. doi: 10.1158/0008-5472.CAN-15-3376

13. Cormerais Y, Massard PA, Vucetic M, Giuliano S, Tambutté E, Durivault J, et al. The glutamine transporter ASCT2 (SLC1A5) promotes tumor growth independently of the amino acid transporter LAT1 (SLC7A5). J Biol Chem. (2018) 293:2877-87. doi: 10.1074/jbc.RA117.001342

14. Ishimoto T, Nagano O, Yae T, Tamada M, Motohara T, Oshima H, et al. CD44 variant regulates redox status in cancer cells by stabilizing the xCT subunit of system xc- and thereby promotes tumor growth. Cancer Cell. (2011) 19:387-400. doi: 10.1016/j.ccr.2011.01.038

15. Müller S, Sindikubwabo F, Cañeque T, Lafon A, Versini A, Lombard B, et al. CD44 regulates epigenetic plasticity by mediating iron endocytosis. bioRxiv [Preprint] (2019). doi: 10.1101/693424

\section{AUTHOR CONTRIBUTIONS}

$\mathrm{BD}$ wrote this review and MV and JP revised it.

\section{ACKNOWLEDGMENTS}

$\mathrm{BD}$ and $\mathrm{MV}$ are founded by the Center Scientifique de Monaco (CSM) and the Monegasque association GEMLUC (Groupement des Entreprises Monégasques dans la Lutte contre le Cancer).

16. Daher B, Parks SK, Durivault J, Cormerais Y, Baidarjad H, Tambutte $\mathrm{E}$, et al. Genetic ablation of the cystine transporter $\mathrm{xCT}$ in PDAC cells inhibits mTORC1, growth, survival, and tumor formation via nutrient and oxidative stresses. Cancer Res. (2019) 79:3877-90. doi: 10.1158/0008-5472.CAN-18-3855

17. Sato H, Kuriyama-Matsumura K, Siow RCM, Ishii T, Bannai S, Mann GE. Induction of cystine transport via system xc- and maintenance of intracellular glutathione levels in pancreatic acinar and islet cell lines. Biochim Biophys Acta. (1998) 1414:85-94. doi: 10.1016/S0005-2736(98)00159-X

18. Sato H, Shiiya A, Kimata M, Maebara K, Tamba M, Sakakura Y, et al. Redox imbalance in cystine/glutamate transporter-deficient mice. J Biol Chem. (2005) 280:37423-9. doi: 10.1074/jbc.M506439200

19. Utsunomiya-Tate N, Endou H, Kanai Y. Cloning and functional characterization of a system ASC-like $\mathrm{Na}+$-dependent neutral amino acid transporter. J Biol Chem. (1996) 271:14883-90. doi: 10.1074/jbc.271.25. 14883

20. Scalise M, Pochini L, Pingitore P, Hedfalk K, Indiveri C. Cysteine is not a substrate but a specific modulator of human ASCT2 (SLC1A5) transporter. FEBS Lett. (2015) 589:3617-23. doi: 10.1016/j.febslet.2015.10.011

21. Condon KJ, Sabatini DM. Nutrient regulation of mTORC1 at a glance. J Cell Sci. (2019) 132:jcs222570. doi: 10.1242/jcs.222570

22. Mossmann D, Park S, Hall MN. mTOR signalling and cellular metabolism are mutual determinants in cancer. Nat Rev Cancer. (2018) 18:744-57. doi: 10.1038/s41568-018-0074-8

23. Yu X, Long YC. Crosstalk between cystine and glutathione is critical for the regulation of amino acid signaling pathways and ferroptosis. Sci Rep. (2016) 6:30033. doi: 10.1038/srep30033

24. Stockwell BR, Friedmann Angeli JP, Bayir H, Bush AI, Conrad M, Dixon SJ, et al. Ferroptosis: a regulated cell death nexus linking metabolism, redox biology, and disease. Cell. (2017) 171:273-85. doi: 10.1016/j.cell.2017. 09.021

25. Maurin A-C, Jousse C, Averous J, Parry L, Bruhat A, Cherasse Y, et al. The GCN2 kinase biases feeding behavior to maintain amino acid homeostasis in omnivores. Cell Metab. (2005) 1:273-7. doi: 10.1016/j.cmet.2005.03.004

26. Dever TE, Hinnebusch AG. GCN2 whets the appetite for amino acids. Mol Cell. (2005) 18:141-2. doi: 10.1016/j.molcel.2005.03.023

27. Ye J, Kumanova M, Hart LS, Sloane K, Zhang H, De Panis DN, et al. The GCN2-ATF4 pathway is critical for tumour cell survival and proliferation in response to nutrient deprivation. EMBO J. (2010) 29:2082-96. doi: 10.1038/emboj.2010.81

28. Cobler L, Zhang H, Suri P, Park C, Timmerman LA. xCT inhibition sensitizes tumors to \&\#x3B3;-radiation via glutathione reduction. Oncotarget. (2018) 9:32280-97. doi: 10.18632/oncotarget.25794

29. Delaunay-Moisan A, Ponsero A, Toledano MB. Reexamining the function of glutathione in oxidative protein folding and secretion. Antioxid Redox Signal. (2017) 27:1178-99. doi: 10.1089/ars.2017.7148

30. Teskey G, Abrahem R, Cao R, Gyurjian K, Islamoglu H, Lucero M, et al. Glutathione as a marker for human disease. Adv Clin Chem. (2018) 87141-59. doi: 10.1016/bs.acc.2018.07.004

31. Bansal A, Simon MC. Glutathione metabolism in cancer progression and treatment resistance. J Cell Biol. (2018) 217:2291-8. doi: $10.1083 /$ jcb. 201804161 
32. Orlowski M, Meister A. $\gamma$-glutamyl-p-nitroanilide: a new convenient substrate for determination and study of 1 - and $d-\gamma$-glutamyltranspeptidase activities. Biochim Biophys Acta. (1963) 73:679-81. doi: 10.1016/0926-6569(63)90197-4

33. Lu JF, Pokharel D, Bebawy M. MRP1 and its role in anticancer drug resistance. Drug Metab Rev. (2015) 47:406-19. doi: 10.3109/03602532.2015.1105253

34. Pompella A, Corti A, Paolicchi A, Giommarelli C, Zunino F. $\gamma$ glutamyltransferase, redox regulation and cancer drug resistance. Curr Opin Pharmacol. (2007) 7:360-6. doi: 10.1016/j.coph.2007.04.004

35. Dringen R, Hamprecht B, Bröer $S$. The peptide transporter pept 2 mediates the uptake of the glutathione precursor cysgly in astroglia-rich primary cultures. J Neurochem. (2002) 71:388-93. doi: 10.1046/j.1471-4159.1998.71010388.x

36. Frey IM, Rubio-Aliaga I, Siewert A, Sailer D, Drobyshev A, Beckers J, et al. Profiling at mRNA, protein, and metabolite levels reveals alterations in renal amino acid handling and glutathione metabolism in kidney tissue of pept2 -/- mice. Physiol Genomics. (2007) 28:301-10. doi: 10.1152/physiolgenomics.00193.2006

37. Yang MS, Chan HW, Yu LC. Glutathione peroxidase and glutathione reductase activities are partially responsible for determining the susceptibility of cells to oxidative stress. Toxicology. (2006) 226:126-30. doi: 10.1016/j.tox.2006.06.008

38. Bannai S, Tateishi N. Role of membrane transport in metabolism and function of glutathione in mammals. J Membr Biol. (1986) 89:1-8. doi: 10.1007/BF01870891

39. Oestreicher J, Morgan B. Glutathione: subcellular distribution and membrane transport. Biochem Cell Biol. (2019) 97:270-89. doi: 10.1139/bcb-2018-0189

40. Sasaki H, Sato H, Kuriyama-Matsumura K, Sato K, Maebara K, Wang H, et al. Electrophile response element-mediated induction of the cystine/glutamate exchange transporter gene expression. J Biol Chem. (2002) 277:44765-71. doi: 10.1074/jbc.M208704200

41. Conrad M, Sato H. The oxidative stress-inducible cystine/glutamate antiporter, system x c - : cystine supplier and beyond. Amino Acids. (2012) 42:231-46. doi: 10.1007/s00726-011-0867-5

42. Dixon SJ, Lemberg KM, Lamprecht MR, Skouta R, Zaitsev EM, Gleason CE, et al. Ferroptosis: an iron-dependent form of nonapoptotic cell death. Cell. (2012) 149:1060-72. doi: 10.1016/j.cell.2012.03.042

43. Yin $\mathrm{H}, \mathrm{Xu} \mathrm{L}$, Porter NA. Free radical lipid peroxidation: mechanisms and analysis. Chem Rev. (2011) 111:5944-72. doi: 10.1021/cr200084z

44. Shirlee Tan BSP, David Schubert BSP, Pamela Maher BSP. Oxytosis: a novel form of programmed cell death. Curr Top Med Chem. (2001) 1:497-506. doi: 10.2174/1568026013394741

45. Bannai S, Ishii T. A novel function of glutamine in cell culture: utilization of glutamine for the uptake of cystine in human fibroblasts. J Cell Physiol. (1988) 137:360-6. doi: 10.1002/jcp.1041370221

46. Okazaki S, Umene K, Yamasaki J, Suina K, Otsuki Y, Yoshikawa M, et al. Glutaminolysis-related genes determine sensitivity to xCT-targeted therapy in head and neck squamous cell carcinoma. Cancer Sci. (2019) 110:3453-63. doi: $10.1111 /$ cas. 14182

47. Luo $\mathrm{M}$, Wu L, Zhang $\mathrm{K}$, Wang $\mathrm{H}$, Zhang $\mathrm{T}$, Gutierrez $\mathrm{L}$, et al. miR-137 regulates ferroptosis by targeting glutamine transporter SLC1A5 in melanoma. Cell Death Differ. (2018) 25:1457-72. doi: 10.1038/s41418-017-0053-8

48. Xu T, Ding W, Ji X, Ao X, Liu Y, Yu W, et al. Molecular mechanisms of ferroptosis and its role in cancer therapy. J Cell Mol Med. (2019) 23:4900-12. doi: $10.1111 / \mathrm{jcmm} .14511$

49. Zhang Y, Tan H, Daniels JD, Zandkarimi F, Liu H, Brown LM, et al. Imidazole ketone erastin induces ferroptosis and slows tumor growth in a mouse lymphoma model. Cell Chem Biol. (2019) 26:623-33.e9. doi: 10.1016/j.chembiol.2019.01.008

50. Ji X, Qian J, Rahman SMJ, Siska PJ, Zou Y, Harris BK, et al. xCT (SLC7A11)mediated metabolic reprogramming promotes non-small cell lung cancer progression. Oncogene. (2018) 37:5007-19. doi: 10.1038/s41388-018-0307-Z

51. Patel D, Kharkar PS, Gandhi NS, Kaur E, Dutt S, Nandave M. Novel analogs of sulfasalazine as system x c- antiporter inhibitors: insights from the molecular modeling studies. Drug Dev Res. (2019) 80:758-77. doi: 10.1002/ddr. 21557

52. Liang C, Zhang X, Yang M, Dong X. Recent progress in ferroptosis inducers for cancer therapy. Adv Mater. (2019) 31:1904197. doi: 10.1002/adma.201904197
53. Cramer SL, Saha A, Liu J, Tadi S, Tiziani S, Yan W, et al. Systemic depletion of L-cyst(e)ine with cyst(e)inase increases reactive oxygen species and suppresses tumor growth. Nat Med. (2017) 23:120-7. doi: 10.1038/n m. 4232

54. Liu N, Lin X, Huang C. Activation of the reverse transsulfuration pathway through NRF2/CBS confers erastin-induced ferroptosis resistance. $\mathrm{Br} \mathrm{J}$ Cancer. (2020) 122:279-92. doi: 10.1038/s41416-019-0660-x

55. Zhu J, Berisa M, Schwörer S, Qin W, Cross JR, Thompson CB. Transsulfuration activity can support cell growth upon extracellular cysteine limitation. Cell Metab. (2019) 30:865-76.e5. doi: 10.1016/j.cmet.2019.09.009

56. Leikam C, Hufnagel A, Walz S, Kneitz S, Fekete A, Müller MJ, et al. Cystathionase mediates senescence evasion in melanocytes and melanoma cells. Oncogene. (2014) 33:771-82. doi: 10.1038/onc.2012.641

57. Griffith OW, Meister A. Potent and specific inhibition of glutathione synthesis by buthionine sulfoximine (S-n-butyl homocysteine sulfoximine). J Biol Chem. (1979) 254:7558-60.

58. Cao JY, Poddar A, Magtanong L, Lumb JH, Mileur TR, Reid MA, et al. A genome-wide haploid genetic screen identifies regulators of glutathione abundance and ferroptosis sensitivity. Cell Rep. (2019) 26:1544-56.e8. doi: 10.1016/j.celrep.2019.01.043

59. Bachhawat AK, Kaur A. Glutathione degradation. Antioxid Redox Signal. (2017) 27:1200-16. doi: 10.1089/ars.2017.7136

60. Chen M-S, Wang S-F, Hsu C-Y, Yin P-H, Yeh T-S, Lee H-C, et al. $\mathrm{CHAC1}$ degradation of glutathione enhances cystine-starvation-induced necroptosis and ferroptosis in human triple negative breast cancer cells via the GCN2-eIF2 $\alpha-\mathrm{ATF}_{4}$ pathway. Oncotarget. (2017) 8:114588-602. doi: 10.18632/oncotarget.23055

61. Wang N, Zeng G-Z, Yin J-L, Bian Z-X. Artesunate activates the ATF4CHOP-CHAC1 pathway and affects ferroptosis in burkitt's lymphoma. Biochem Biophys Res Commun. (2019) 519:533-9. doi: 10.1016/j.bbrc.2019. 09.023

62. Shen L, Qi W, Bai J, Zuo C, Bai D, Gao W, et al. Resibufogenin (RB) inhibited colorectal cancer cell growth and tumorigenesis through triggering ferroptosis and ROS production mediated by GPX4 inactivation. Anat Rec. (2020). doi: 10.1002/ar.24378. [Epub ahead of print].

63. Hangauer MJ, Viswanathan VS, Ryan MJ, Bole D, Eaton JK, Matov A, et al. Drug-tolerant persister cancer cells are vulnerable to GPX4 inhibition. Nature. (2017) 551:247-50. doi: 10.1038/nature24297

64. Yoo S-E, Chen L, Na R, Liu Y, Rios C, Van Remmen H, et al. Gpx4 ablation in adult mice results in a lethal phenotype accompanied by neuronal loss in brain. Free Radic Biol Med. (2012) 52:1820-7. doi: 10.1016/j.freeradbiomed.2012.02.043

65. Dalton TP, Dieter MZ, Yang Y, Shertzer HG, Nebert DW. Knockout of the mouse glutamate cysteine ligase catalytic subunit (Gclc) gene: embryonic lethal when homozygous, and proposed model for moderate glutathione deficiency when heterozygous. Biochem Biophys Res Commun. (2000) 279:324-9. doi: 10.1006/bbrc.2000.3930

66. Doll S, Freitas FP, Shah R, Aldrovandi M, da Silva MC, Ingold I, et al. FSP1 is a glutathione-independent ferroptosis suppressor. Nature. (2019) 575:693-8. doi: 10.1038/s41586-019-1707-0

67. Bersuker K, Hendricks JM, Li Z, Magtanong L, Ford B, Tang PH, et al. The CoQ oxidoreductase FSP1 acts parallel to GPX4 to inhibit ferroptosis. Nature. (2019) 575:688-92. doi: 10.1038/s41586-019-1705-2

68. Doll S, Proneth B, Tyurina YY, Panzilius E, Kobayashi S, Ingold I, et al. ACSL4 dictates ferroptosis sensitivity by shaping cellular lipid composition. Nat Chem Biol. (2017) 13:91-8. doi: 10.1038/nchembio.2239

69. Badgley MA, Kremer DM, Maurer HC, DelGiorno KE, Lee H-J, Purohit V, et al. Cysteine depletion induces pancreatic tumor ferroptosis in mice. Science. (2020) 368:85-9. doi: 10.1126/science.aaw9872

70. Magtanong L, Ko PJ, Dixon SJ. Emerging roles for lipids in non-apoptotic cell death. Cell Death Differ. (2016) 23:1099-109. doi: 10.1038/cdd.2 016.25

71. Lewis-Wambi JS, Kim HR, Wambi C, Patel R, Pyle JR, Klein-Szanto AJ, et al. Buthionine sulfoximine sensitizes antihormone-resistant human breast cancer cells to estrogen-induced apoptosis. Breast Cancer Res. (2008) 10:R104. doi: $10.1186 /$ bcr 2208

72. Ramos AM, Aller P. Quercetin decreases intracellular GSH content and potentiates the apoptotic action of the antileukemic drug arsenic trioxide 
in human leukemia cell lines. Biochem Pharmacol. (2008) 75:1912-23. doi: 10.1016/j.bcp.2008.02.007

73. Lv H, Zhen C, Liu J, Yang $\mathrm{P}$, Hu L, Shang P. Unraveling the potential role of glutathione in multiple forms of cell death in cancer therapy. Oxid Med Cell Longev. (2019) 2019:3150145. doi: 10.1155/2019/31 50145

74. Harris IS, Endress JE, Coloff JL, Selfors LM, McBrayer SK, Rosenbluth JM, et al. Deubiquitinases maintain protein homeostasis and survival of cancer cells upon glutathione depletion. Cell Metab. (2019) 29:1166-81.e6. doi: 10.1016/j.cmet.2019. 01.020
Conflict of Interest: The authors declare that the research was conducted in the absence of any commercial or financial relationships that could be construed as a potential conflict of interest.

Copyright (๑ 2020 Daher, Vučetić and Pouysségur. This is an open-access article distributed under the terms of the Creative Commons Attribution License (CC BY). The use, distribution or reproduction in other forums is permitted, provided the original author(s) and the copyright owner(s) are credited and that the original publication in this journal is cited, in accordance with accepted academic practice. No use, distribution or reproduction is permitted which does not comply with these terms. 